\title{
Fabrication of photonic band-gap crystals
}

\author{
C. C. Cheng and A. Scherer \\ California Institute of Technology, Pasadena, California 91125
}

(Received 19 June 1995; accepted 9 August 1995)

\begin{abstract}
We describe the fabrication of three-dimensional photonic crystals using a reproducible and reliable procedure consisting of electron beam lithography followed by a sequence of dry etching steps. Careful fabrication has enabled us to define photonic crystals with $280 \mathrm{~nm}$ holes defined with 350 $\mathrm{nm}$ center to center spacings in GaAsP and GaAs epilayers. We construct these photonic crystals by transferring a submicron pattern of holes from 70-nm-thick polymethylmethacrylate resist layers into 300-nm-thick silicon dioxide ion etch masks, and then anisotropically angle etching the III-V semiconductor material using this mask. Here, we show the procedure used to generate photonic crystals with up to four lattice periods depth. () 1995 American Vacuum Society.
\end{abstract}

\section{INTRODUCTION}

A photonic band-gap crystal is a three-dimensional dielectric structure in which electromagnetic waves are forbidden irrespective of their propagation directions. ${ }^{1-5}$ Both computer models and microwave measurements have shown that a face centered cubic (fcc) crystal of holes in a dielectric matrix exhibits such a photonic band gap. ${ }^{6}$ These threedimensionally periodic dielectric structures are frequently called "photonic crystals." We have developed the technology for making valuable photonic crystals and use a combination of high resolution electron beam lithography and anisotropic ion beam etching to define such crystals in compound semiconductors. A hexagonal hole array etch mask is defined by electron beam lithography and then angle etched into the semiconductor wafer by chemically assisted ion beam etching (Fig. 1).

Photonic crystals will be useful as electromagnetic microcavities for single-mode light emitting diodes. These structures are expected to exhibit inhibited spontaneous emission, which may lower the power requirements and increase the reliability of light emitters, particularly of optical arrays. ${ }^{8}$ Photonic crystals can also show enhanced spontaneous emission which would allow faster modulation speeds for optical interconnects. Another technologically important application of photonic band-gap structures lies in microfabricating high reflectivity mirrors in materials systems where epitaxial growth of mirrors is difficult because of the low inherent refractive index contrast between lattice-matched materials. In these semiconductors, photonic crystals can be microfabricated to define three-dimensional (3D) mirrors with geometrically controlled wavelengths and wide free spectral ranges. The 3D interlinked mesh structure which results from microfabricating a fcc crystal is ideally suited for electrical pumping of optoelectronic devices and for directionally coupling out light emission.

The key to constructing such a useful optical crystal in the visible wavelength range lies in constructing a durable etch mask defining a hexagonal pattern of submicron holes and subsequent anisotropic angle etching this mask into the material of interest. ${ }^{8}$ Here, we show the processing details which we have developed to microfabricate photonic bandgap crystals.

\section{PROCEDURE}

We use the combination of wet and dry etching technologies ${ }^{9}$ to fabricate $3 \mathrm{D}$ photonic band-gap structures in the optical wavelength range. Our lithographic process is schematically summarized in Fig. 2. First, electron beam lithography with a $30 \mathrm{kV}$ incident beam energy is used to create a hexagonal hole pattern on the surface of the sample, which corresponds to the (111) plane of the microfabricated fcc crystal. These lithographic patterns are defined over square areas of $50 \times 50 \mu \mathrm{m}^{2}$ on a spun-on 70-nm-thick resist layer of high molecular weight polymethylmethacrylate (PMMA). The beam-written pattern is developed for $15 \mathrm{~s}$ in a 3:7 mixture of cellusolve in methanol. Next, we transfer the PMMA patterns into a 35-nm-thick gold layer by argon ion milling. Then, we use the gold layer as a mask to wet chemically etch $40 \mathrm{~nm}$ Cr in a commercial chrome mask etch solution. To further improve the mask durability, we transfer the chromium pattern into $300 \mathrm{~nm}$ of sputter-deposited silicon dioxide by reactive ion etching using $30 \mathrm{mTorr}^{\mathrm{C}_{2} \mathrm{~F}_{6} \text { as }}$ the reactive gas at an etch rate of $15 \mathrm{~nm} / \mathrm{min}$. Finally, we use a chemically assisted ion beam etching (CAIBE) system ${ }^{10}$ to fabricate the optical crystals in GaAs or GaAsP by angle etching of the masked semiconductor with an argon ion beam assisted by $\mathrm{Cl}_{2}$ reactive gas. During this etch, the surface of the semiconductor is tilted at $35^{\circ}$ to the normal and in the sample is rotated into the three directions which correspond to the close-packed $\langle 110\rangle$ directions of the microfabricated fcc crystal. In this way, a 3D fcc photonic crystal is generated. Provided that the pattern transfer is anisotropic, the lithographic dimensions obtained during electron beam exposure, together with the etch depth, determine the optical response of the crystal. This mirror characteristics are measured by transmission experiments for which the photonic band-gap crystals are placed into $50-\mu \mathrm{m}$-diam microfabricated nickel apertures and their transmission spectra are determined. These measured optical results are then compared to measurements of microwave models of photonic band-gap structures. $^{8}$ 


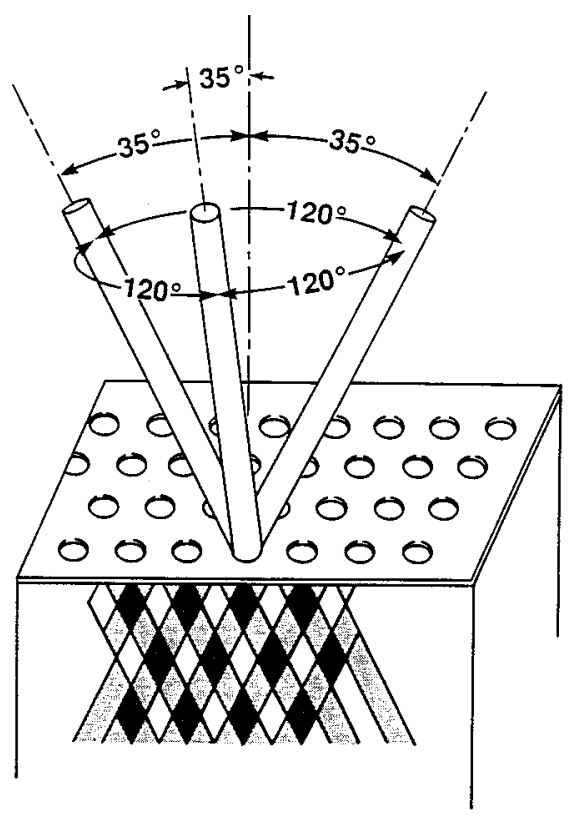

FIG. 1. Schematic figure illustrating the structure of the fcc photonic bandgap crystal. The rods shown in this figure correspond to the three $\langle 110\rangle$ directions which are etched during the CAIBE process, and the surface of the crystal corresponds to the (111) plane of the microfabricated fcc crystal.

\section{RESULTS}

\section{A. Optimization of the mask fabrication sequence}

To optimize the fabrication of three-dimensional structures such as photonic crystals, it is extremely important to accurately characterize the quality and the depth of the structures during all of the steps in the processing sequence. In submicron patterns, this inspection is most conveniently accomplished by using a scanning electron microscope. Figures 3(a) and 3(b) are electron micrographs taken during the etch mask fabrication after ion etching of the gold mask and after reactive ion etching of the silicon dioxide mask, respectively. From these scanning electron microscopy (SEM) micrographs, we observe that the high resolution of the original electron beam resist pattern does not deteriorate significantly during the pattern transfer into the thick silicon dioxide layer.

Angle etching with a single planar mask layer requires careful selection of the mask material and the ion etching conditions in order to maximize the selectivity between the semiconductor etch rate and the mask erosion rate since the mask must be thin. Thin masks are necessary to minimize shadowing of the nominally open material by the etch mask during the angle etch (shown in Fig. 2), which would result in elliptically shaped holes with small sizes. To obtain three period deep photonic crystals, we calculate that the etch rate selectivities required have to be above 15:1. This mask selection is further complicated by the lower etch rates measured when defining small via-holes compared to large open surfaces, making accurate etch rate measurements difficult.

\section{B. Pattern transfer by CAIBE}

High etch rate selectivities are usually obtained when the semiconductor reactivity of the etch is increased. When etch-
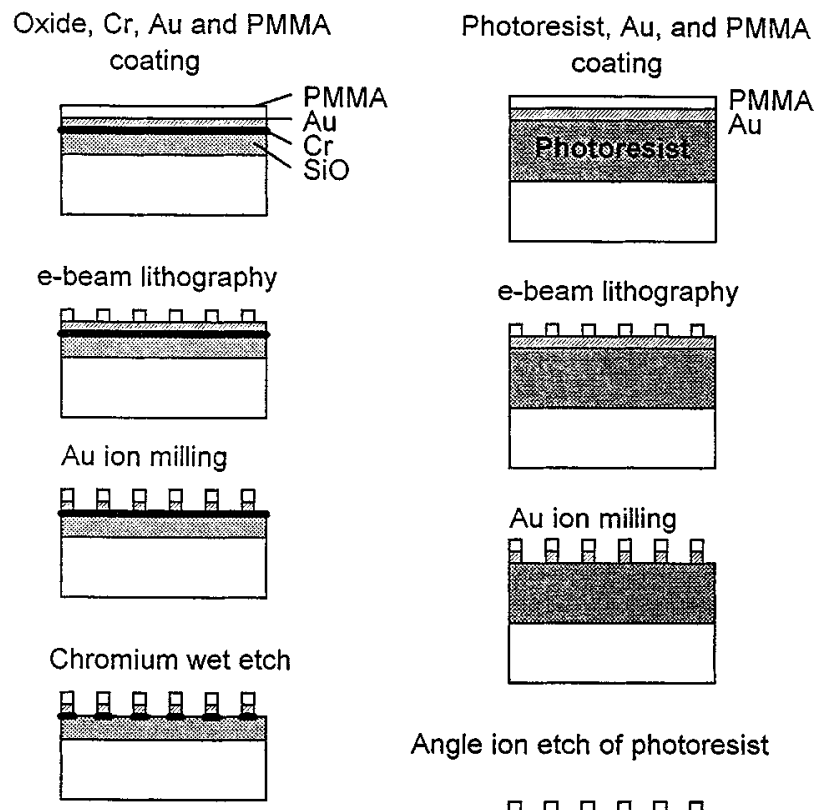

Oxide reactive ion etch

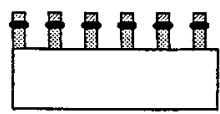

Angle ion etch of semiconductor

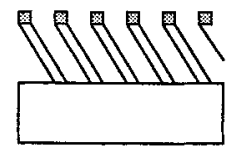

Angle ion etch of photoresist

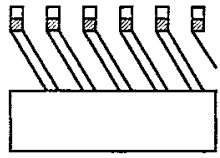

Angle ion etch of semiconductor

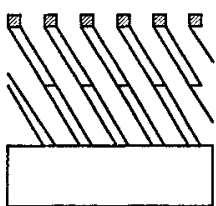

FIG. 2. Schematic description of the fabrication procedures presently used to generate photonic band-gap crystals, and planned to be used for deeper structures.

ing large structures, this is accomplished either by increasing the surface temperature or the reactive gas pressure. However, isotropic etching and mask undercut results from both of these cases, hollowing out the structure underneath the etch mask, as shown in Fig. 4(a). The extent of the undercut is revealed by cleaving the semiconductor wafer through the photonic crystal. Therefore, the etching system is optimized to maximize the surface etch rate without undercutting the mask, and it is important to hold constant the temperature and the reactive gas pressure during the CAIBE process. ${ }^{11}$ Cleaved cross-sectioning also allows the determination of the relative etch depths of the holes which define the three $\langle 110\rangle$ directions. Etching of the first etched hole removes more than $50 \%$ of the semiconductor material, and thus the second and third holes require correspondingly less etching time to define a crystal with a given depth. Careful calibration of the etching times of all three holes is required in order to obtain a uniform photonic crystal with a given depth. Although the structure in Fig. 4(b) is anisotropically etched with smooth sidewalls in all three directions, the variations of the relative depth compromises the optical response of the resulting photonic band-gap mirror. Microscopic roughness of the CAIBE 


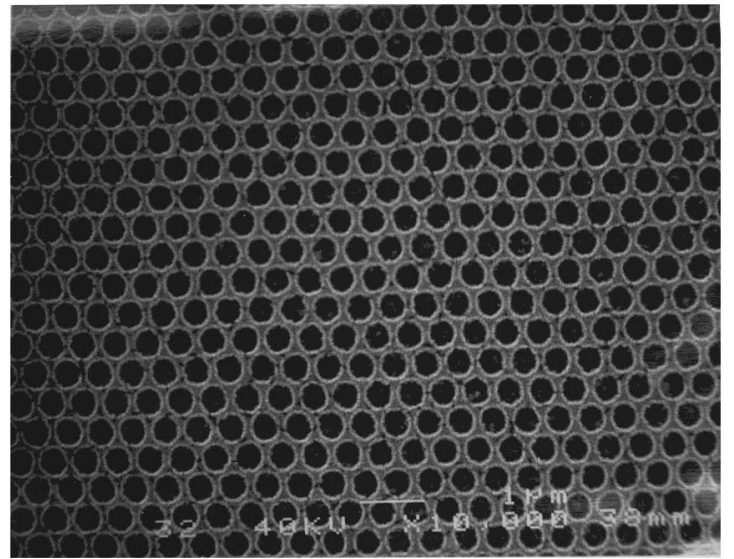

(a)

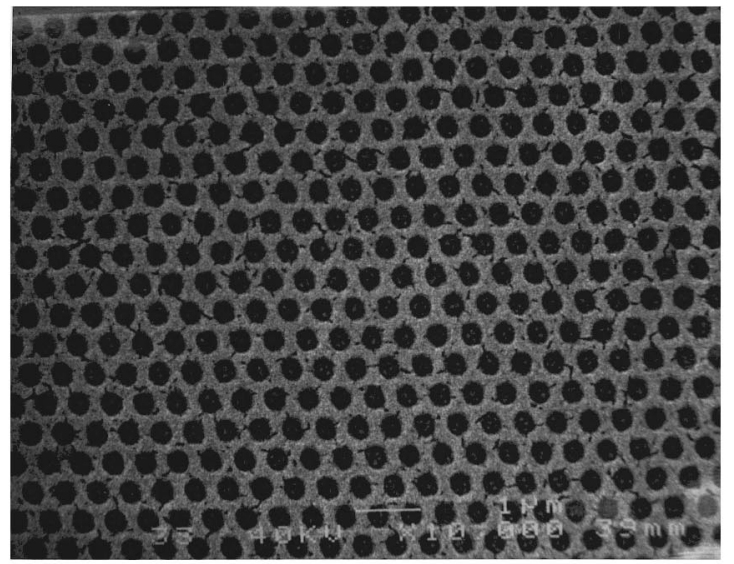

(b)

FIG. 3. (a) SEM micrograph showing the lithographically defined hole mask after gold ion milling. (b) SEM micrograph of the etch mask after transferring into the silicon dioxide layer.

process also influences the quality of the band-gap structure, since such roughness results in a blurring of the refractive index modulation, and hence gives rise to lower measured reflectivities. In Fig. 4(c), we show an example of this microscopic roughness in an SEM micrograph.

\section{Process reliability and limitations}

When the CAIBE process is optimized, we can obtain high quality crystals, such as the ones shown in Figs. 5(a) and 5(b). Here, we show photonic band-gap structures which have been defined three layers deep into a GaAs crystal, by etching a pattern of $400 \mathrm{~nm}$ holes spaced $520 \mathrm{~nm}$ from center to center. This crystal was defined in a microfabricated aperture for optical measurement, and subsequently cleaved to reveal the three-dimensional nature of the crystal. Since these photonic crystals exhibit $84 \%$ porosity, the etch depth of optimally etched structures can also be determined nondestructively by slightly tilting the surface of the patterned semiconductor, and observation of the number of repeating features underneath the surface in the SEM. Figure 6(a) shows how this method can be used on a GaAs photonic crystal which was etched four lattice periods deep. This inspection also allows the measurement of the hole sizes, hole

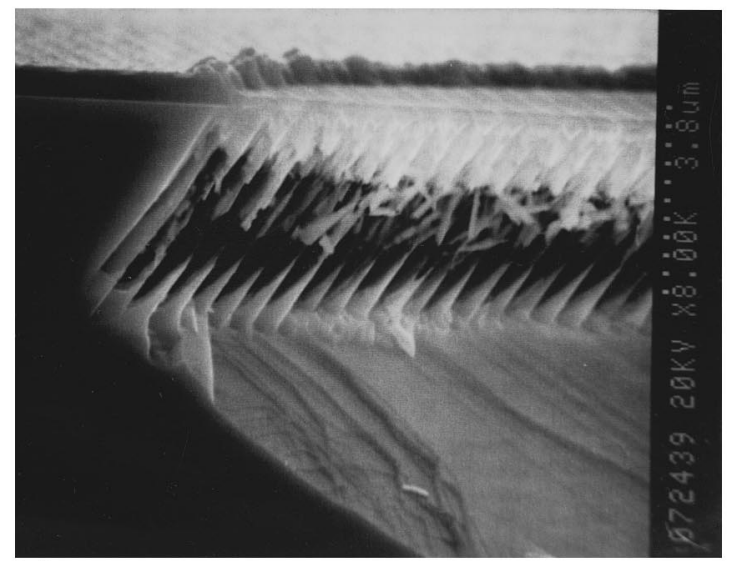

(a)

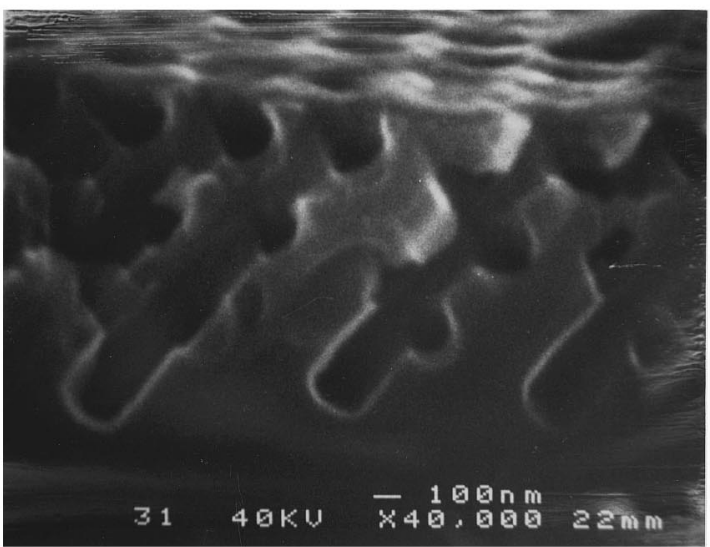

(b)

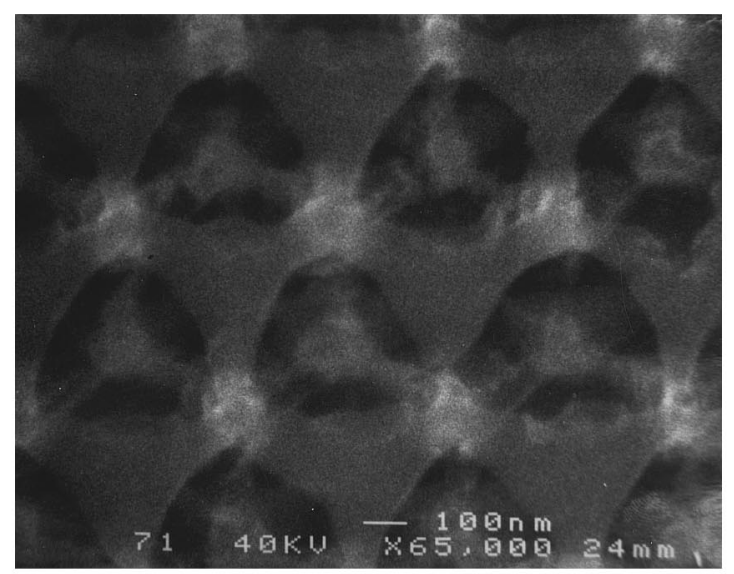

(c)

FIG. 4. (a) SEM micrograph of an undercut photonic band-gap mask after etching with too high of a chlorine gas pressure. (b) SEM micrograph of a photonic crystal in which the etching time used for defining the three $\langle 110\rangle$ directions is not properly balanced. (c) Microscopic surface roughness shown on a photonic crystal. This results from too high of an etch rate.

spacings, and porosities. A similar inspection of our smallest etched photonic band-gap crystal in GaAsP diode material reveals that the crystal is defined by 280 -nm-diam holes with $350 \mathrm{~nm}$ center to center distance and a depth of three lattice periods [Fig. 6(b)]. This crystal was optimized for a center wavelength in the reflectivity spectrum of $700 \mathrm{~nm}$, which matches the luminescence peak of the GaAsP crystal. We 


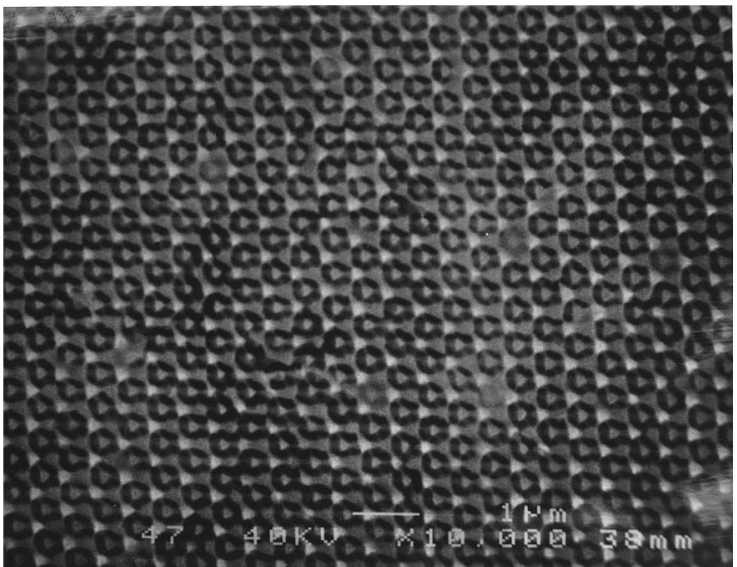

(a)

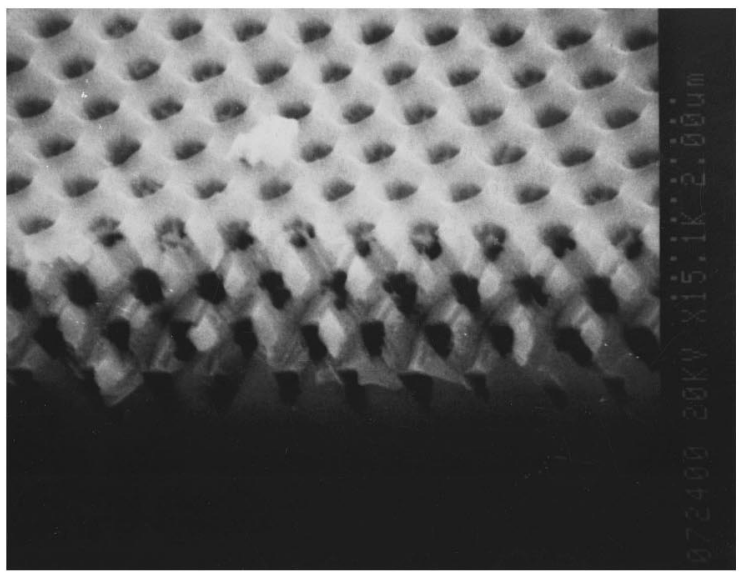

(b)

FIG. 5. (a) Cross-sectional view of a photonic band-gap crystal etched to three lattice periods deep into a GaAs crystal. (b) SEM micrograph taken at normal incidence to the surface of the GaAs wafer showing the (111) surface of a photonic crystal.

expect that six periods of photonic crystal are necessary to obtain a high enough reflectivity to serve as a suitable mirror for a lasing structure. ${ }^{12}$

We have evaluated the processing sequence for reproducibility and reliability of photonic crystal fabrication. The evolution of the processing sequence is schematically summarized in Fig. 2. For structures with up to six periods depth, we expect to define this 3D mask by using a planar etch mask. However, as deeper photonic crystals are required, 3D etch masks have to be developed. We are now pursuing a fabrication technique in which we use oxygen ion etching to define an optical crystal structures template in hard-baked photoresist, which will then be transferred into the substrate material of interest (Fig. 2).

\section{DISCUSSION AND CONCLUSIONS}

In this paper, we demonstrate the methodology required to accomplish the high resolution microfabrication and pattern transfer procedure for making photonic crystals in both GaAs and GaAsP. So far, we have fabricated and characterized two lattice period thick microfabricated photonic bandgap structures with center wavelengths in the visible and near-infrared wavelength range. The diameter of the holes

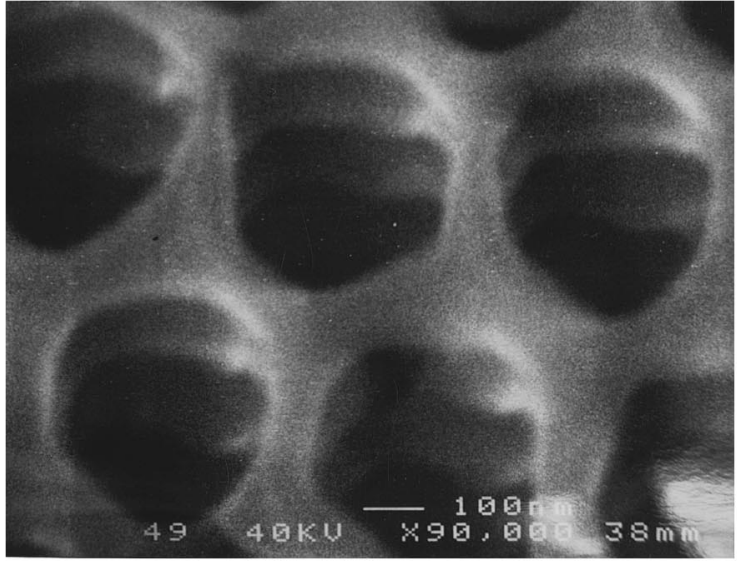

(a)

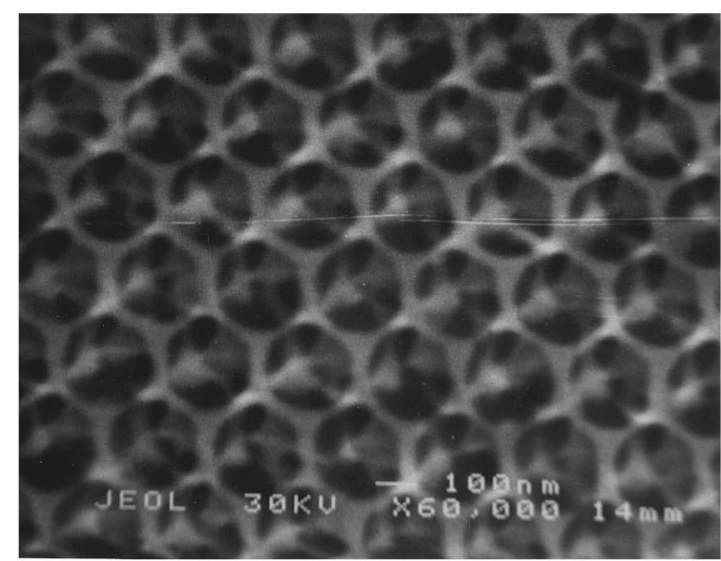

(b)

FIG. 6. (a) Four lattice periods deep photonic crystal illustrated by tilting the crystal $30^{\circ}$ in a SEM. (b) SEM micrograph of three period deep photonic crystal in GaAsP tilted $5^{\circ}$. The hole size is $280 \mathrm{~nm}$ and the spacing is 350 nm center to center.

ranges from 280 to $400 \mathrm{~nm}$, and the distance between holes ranges from 350 to $520 \mathrm{~nm}$. The center frequency of the photonic band-gap mirror can be adjusted lithographically during the mask definition process, and can be as low as 700 $\mathrm{nm}$.

Presently, we are focusing our efforts towards measuring the optical response of our photonic crystals. We have developed the optical transmission measurement techniques which allow us to test these structures through microfabricated metal apertures. A measurement system, based on an optical spectrum analyzer, has been assembled and tested on more conventional mirror structures. We have fabricated several crystals both in $\mathrm{GaAs}$ and $\mathrm{GaAsP} / \mathrm{GaP}$ materials for such measurements.

By producing a photonic band-gap crystal in the optical wavelength range, it is also possible to define ultrasmall optical cavities, which can be used for creating single mode diodes from semiconductor laser material. ${ }^{13}$ Such photonic band-gap devices, which are expected to exhibit high spontaneous emission factors, will be constructed by deliberately microfabricating defects into the photonic crystal and thereby produce acceptor and donor levels, analogous to 3D Fabry-Pérot cavities. ${ }^{12}$ 


\section{ACKNOWLEDGMENTS}

This work was supported by the National Science Foundation under Grant No. ECS-9310681. The authors also gratefully acknowledge the help of Professor Eli Yablonovich and Vincent Arbet-Engels in this work, as well as helpful suggestions from Reynold Johnson and Andrew Cleland.

${ }^{1}$ S. John, Phys. Rev. Lett. 58, 2486 (1987).

${ }^{2}$ S. L. McCall, P. M. Platzman, R. Dalichaouch, D. Smith, and S. Schultz, Phys. Rev. Lett. 67, 2017 (1991).

${ }^{3}$ K. M. Leung and Y. F. Liu, Phys. Rev. Lett. 65, 2646 (1990).

${ }^{4}$ S. L. McCall, A. F. J. Levi, R. E. Slusher, S. J. Pearton, and R. A. Logan, Appl. Phys. Lett. 60, 289 (1992).
${ }^{5}$ E. Yablonovitch and T. J. Gmitter, Phys. Rev. Lett. 63, 1950 (1989).

${ }^{6}$ E. Yablonovitch, T. J. Gmitter, and K. M. Leung, Phys. Rev. Lett. 67, 2295 (1991).

${ }^{7}$ K. M. Ho, C. T. Chan, and C. M. Soukoulis, Phys. Rev. Lett. 65, 3152 (1990).

${ }^{8}$ E. Yablonovitch, Phys. Rev. Lett. 58, 2059 (1987).

${ }^{9}$ R. E. Howard, H. G. Craighead, L. D. Jackel, P. M. Mankiewich, and M. Feldman, J. Vac. Sci. Technol. B 1, 1101 (1983).

${ }^{10}$ A. Scherer, J. L. Jewell, and J. P. Haribson, Opt. Phot. News 2, 9 (1991).

${ }^{11}$ B. P. Van der Gaag and A. Scherer, Appl. Phys. Lett. 56, 481 (1989).

${ }^{12}$ J. L. Jewell, J. P. Harbison, A. Scherer, Y. H. Lee, and L. T. Florez, IEEE J. Quantum Electron. QE-27, 1332 (1991).

${ }^{13}$ E. Yablonovitch, J. Opt. Soc. Am. B 10, (1993). 